\title{
A New Program Design Developed for AC Load Flow Analysis Problems
}

\section{Celal YASAR ${ }^{1}$, Serdar ÖZYÖN ${ }^{2 *}$, Hasan TEMURTAS ${ }^{3}$}

${ }^{1,2}$ Dumlupinar University, Faculty of Engineering, Electrical and Electronics Engineering ${ }^{3}$ Dumlupinar University, Faculty of Engineering, Computer Engineering

\section{Abstract}

The Newton-Raphson method is generally used for iterative solutions of power systems in load flow analysis. This study focuses on a new program structure designed to solve large size power systems accurately and quickly using AC load flow analysis technique. This program structure will provide both easy and accurate data input in solutions of high-capacity data input problems. The data required for solving the problem at hand was obtained from an Excel, and coded in Matlab R2015b and solutions were printed onto a solution file.

\section{Key Words}




\section{INTRODUCTION}

With the increasing demand for electricity, the planning and optimal operation of power generation systems have recently become a very important issue. In order to carry out these operations, it is necessary to analyze AC load flow in power systems. In order to perform AC load flow analysis of a power system, net active and reactive powers of all buses and voltage magnitude and angle of the slack bus should be input into the system. If there are voltage-controlled buses in the system, they should be introduced to the system as buses, the voltage amplitudes of which will be kept constant. The power flow solution provides the voltages, amplitudes and phase angles of all buses. Afterwards, active and reactive powers of the slack, power flows and line losses are calculated.

There are many studies on AC load flow analysis in the literature. While some of these studies deal with newly developed methods, some others focus on interface designs to make a given program easier to use. The latter address load flow analysis using a modified Newton-Raphson method (Panosyan et al., 2004), use of probability techniques for AC load flow analysis (Allan et al., 1977), (Dagur et al., 2014), linearized AC load flow applications (Rosoni et al, 2016) and use of evolutionary computation methods in load flow solutions (Revanthi, 2008).

This study used the Newton-Raphson method for load flow analysis. When using this method, it is very important that problem data are input correctly into load flow programs. If data are input incorrectly, it is unlikely that solutions will be correct. Therefore, data input becomes very important, especially in high-dimensional systems. For this reason, this study focused on a new data input design. Data was extracted from an Excel file and coded, and the program software was run using Matlab R2015b. The system used as a sample application consists of 118 buses and 54 generators.

This paper summarizes the load flow method, provides information on the software developed to provide ease of use, and presents the solution to a multi-dimensional sample system to demonstrate the advantage of the software.

\section{LOAD FLOW ANALYSIS}

Load flow refers to determination of the best mode of operation of existing power systems and calculation of voltage magnitude and phase angle of each bus in a power system. Once the information on buses has been obtained, active and reactive power flows and transmission line losses that occur in transmission lines in the system are calculated (Wood et al., 2013), (Kothari \& Dhillon, 2007), (Özyön,2009).

In power flow in a power system, net active and reactive powers for all buses except one bus are determined. Net power (active $\mathrm{Pk}$ and reactive $\mathrm{Qk}$ ) is equal to the difference between the power supplied to the system and the power drawn from the system, which means that it is the difference between the power supplied by the generator and the power the load consumes depending on the bus. If there is a voltage-controlled bus in the system, the bus voltage magnitude that will be kept constant at this bus should be determined. The generation of the bus-dependent reactive power generator and the bus voltage angle are calculated at the end of power flow solution (Wood et al., 2013), (Kothari \& Dhillon, 2007), (Özyön,2009).

Buses in a power system are divided into various types as load bus, voltage-controlled bus and slack bus. A system has only one slack bus.

Each bus $k$ in a system is defined by active power $P_{k}$, reactive power $Q_{k}$, voltage magnitude $\left|V_{k}\right|$ and voltage angle $\delta_{k}$. Depending on each bus $k$, two of $P_{k}, Q_{k}, \delta_{k}$ and $\left|V_{k}\right|$ values are known and the other two are calculated. The purpose of power flow solutions is to find the two unknown values when the difference between the values of $P_{k}$ and $Q_{k}$ and the calculated values are approximated to zero (Wood et al., 2013).

In power flow studies, bus voltage magnitudes $|\mathrm{Vk}|$ and angles $\delta \mathrm{k}$ are unknown parameters expect for slack buses. They are, mathematically, independent variables, as their values determine the state of a system. Therefore, a power flow problem can be defined as the determination of values of all state variables using equal number of power flow equations based on input data. Once the values of state variables have been calculated, the entire state of the system is known and all values depending on the state variables can be calculated.

\section{DESIGNED PROGRAM AND DATA INPUT}

This study focused on designing a new program that facilitates data input in the load flow program. Data input was performed using Excel, and transferred to Matlab R2015b. The load flow solution was obtained by forming the coding of the program as distinct subprograms and results were printed to a file. The data input sheets in Excel are given in Figures 1, 2 and 3. Power base $\left(S_{\text {base }}\right)$, voltage base $\left(V_{\text {base }}\right)$ and impedance base $\left(Z_{\text {base }}\right)$ values used in the system solution are entered in the Baz_Deg sheet in Figure 1. 


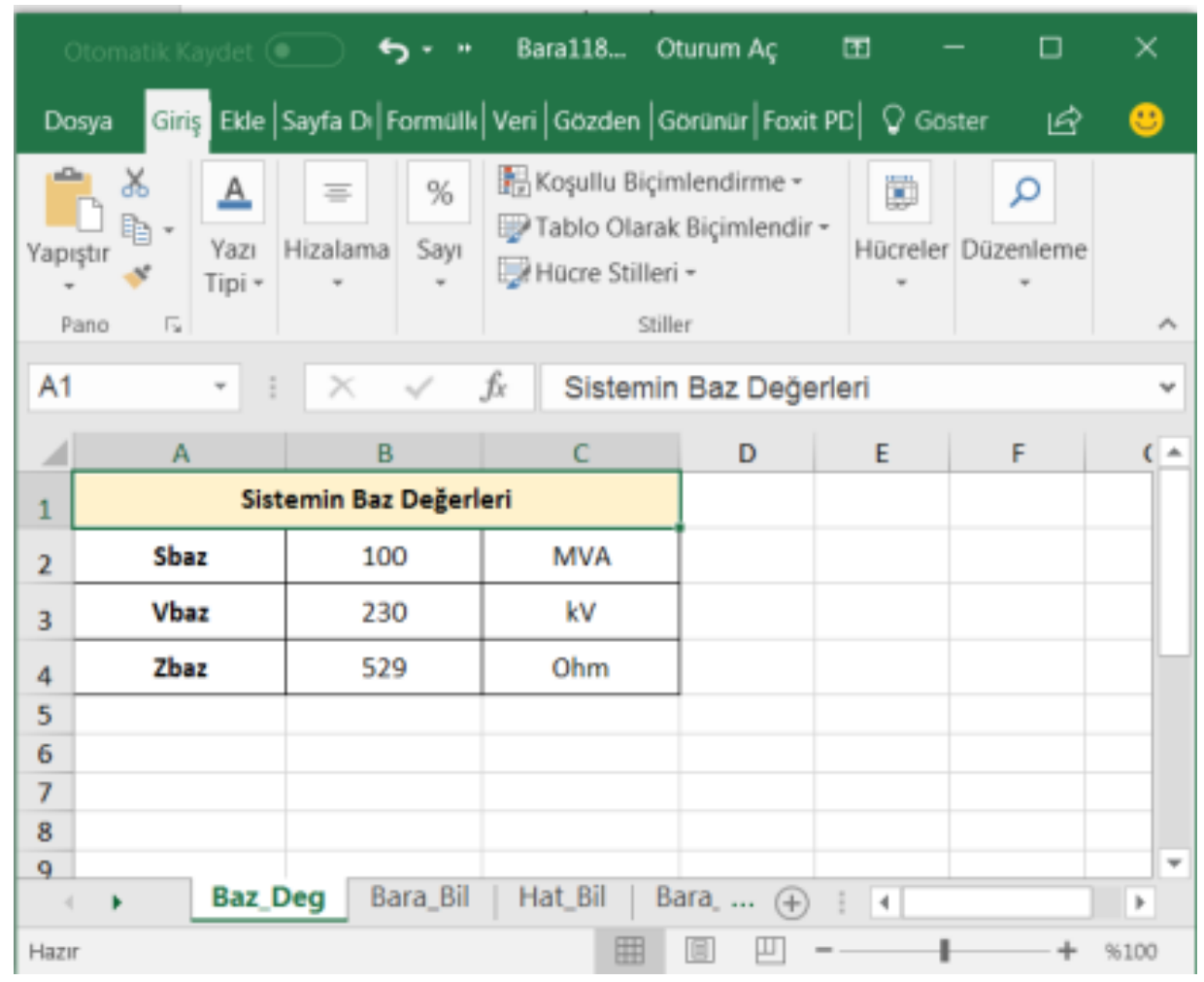

Figure 1. System base values input sheet

The bus numbers, generator bus types (UreTip), bus types (BusTip), active power generation limits of generators $\left(P_{\min }, P_{\max }\right)$, initial value of active power generation $\left(P_{G e n}\right)$, reactive power generation limits of generators $\left(Q_{\min }, Q_{\max }\right)$, initial value of reactive power generation $\left(Q_{G e n}\right)$, active and reactive load values at buses $\left(P_{\text {load }}, Q_{\text {load }}\right)$, voltage limits of buses $\left(V_{\min }\right.$, $\left.V_{\max }\right)$, voltage values of voltage-controlled buses $\left(V_{\text {bus }}\right)$ and bus angles $(A c ̧ \imath)$ used in the solution of the problem are entered in the Bus_Bil sheet given in Figure 2.

The line numbers, serial impedance between lines $(R)$, parallel admittance $(X)$, capacitance values $(B)$, transformer transfer ratio (Tap), transmission line carrying capacities $\left(S_{\text {limit }}\right)$ and maximum angle difference between buses $(M a x A f)$, which are the transmission line information of the system, are entered in the Hat_Bil sheet given in Figure 3.

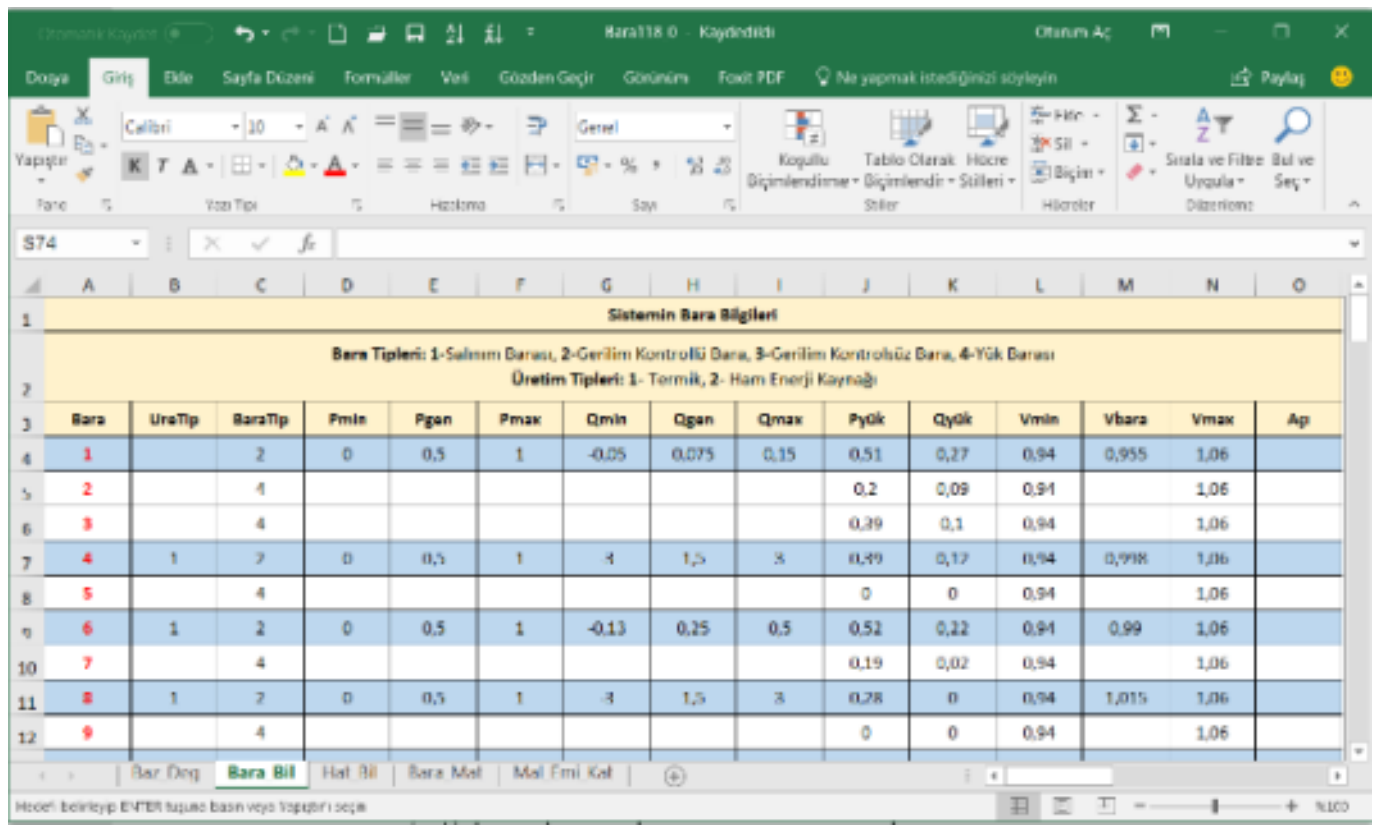

Figure 2. System bus information input sheet 


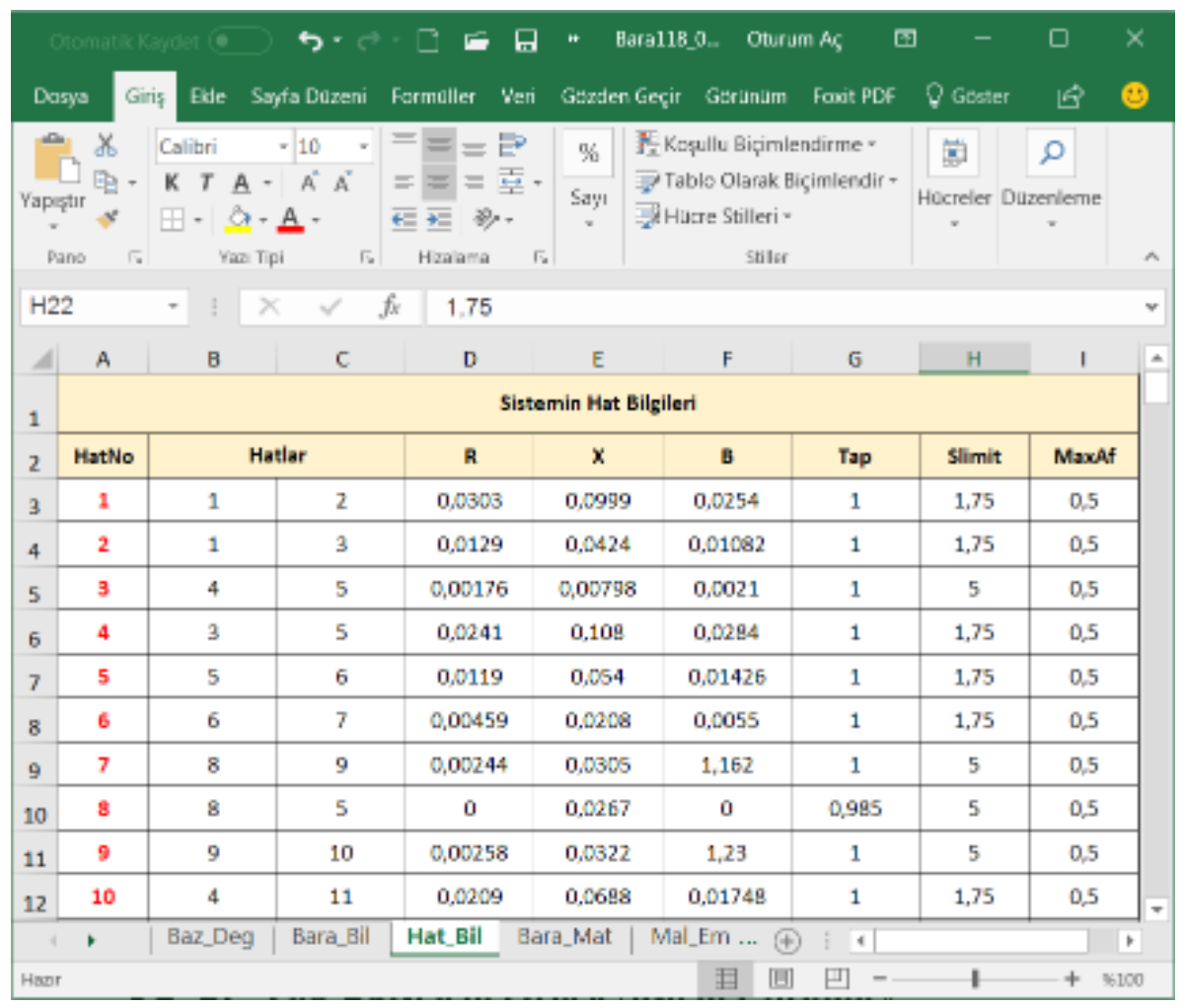

Figure 3. System line information input sheet

In this design used to input system data, the program can easily be integrated into the new state by adding a sheet or column to the data file for additional information required for a new problem or a new system.

After the system data is uploaded to an Excel file, the following code block generated in Matlab is used to convert the data into a matrix form with a .mat extension. The data converted into a matrix form is uploaded to the Matlab application and their load flow is performed and results are printed.

\section{SAMPLE SYSTEM SOLUTION}

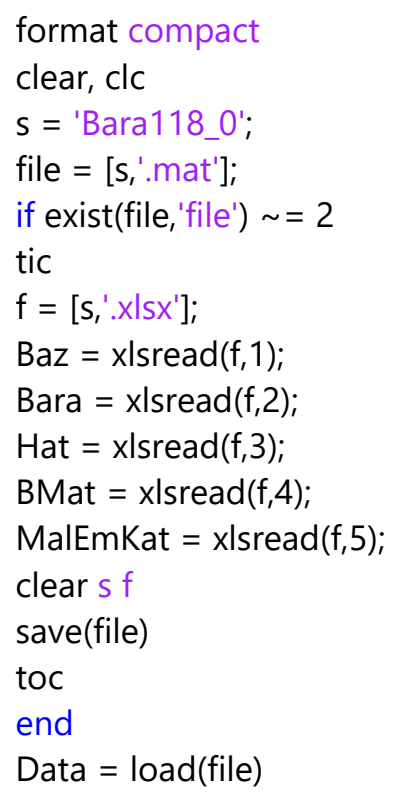


A sample power system consisting of IEEE 118 buses, 54 thermal power generation units and 179 transmission lines referred to as single-line diagram in Appendix Figure 1 was selected in order to test the load flow analysis in this section (Power Systems Test Case Archieve, 2017), (MatPower, 2017), (Zahlay, 2016).

This system is one of the high dimensional systems in the literature. Bus no 69 in the system is a slack bus and its voltage is $1.035 \angle 0^{\circ} \mathrm{pu}$. All 54 generators in the system consist of voltage-controlled buses. 9 transmission lines in the system have transformers. The base values of the system were designated as $S_{\text {base }}=100 \mathrm{MVA}, U_{\text {base }}=230 \mathrm{kVA}$ and $Z_{\text {base }}=529 \mathrm{Ohm}$.

Appendix Table 1 shows the serial impedance, parallel admittance, capacitance values, transformer transfer ratios and line carrying capacities of the nominal $\pi$ equivalent circuits of the transmission lines in the sample system. 91 buses and their active and reactive load values that remain unchanged at a period of time in the system are given as $p u$ in Appendix Table 2. Appendix Table 3 presents the initial values of the load flow of the generation units. The 54 generators in the system are voltage-controlled buses and the bus voltages of these buses are kept constant at the values given in Appendix Table 3. The working limit values of the generation units in the system are given in Appendix Table 4 (Power Systems Test Case Archieve, 2017), (MatPower,2017), (Zahlay, 2016)

The bus voltage magnitude and angle values $\left(V_{k}, \delta_{k}\right)$, active and reactive power generation values $\left(P_{k}, Q_{k}\right)$ as $p u$ in the generation units and transmission line losses obtained from AC load flow in the sample system are given in Annex Table 5. The transmission line losses that occured as a result of AC load flow in the sample system were $P_{\text {loss }}=0,614601 \mathrm{pu}$. The voltage magnitudes of the buses in the system ranged from 0.94 to $1.06 \mathrm{pu}$. The voltage profile of all the buses showing this state is given in Figure 4. The load flow solution time of the large size sample system at the work statiton with Intel Xeon E5-2637 v4 3.50 GHz processor and 128 GB RAM memory was $0.0719492 \mathrm{sec}$.

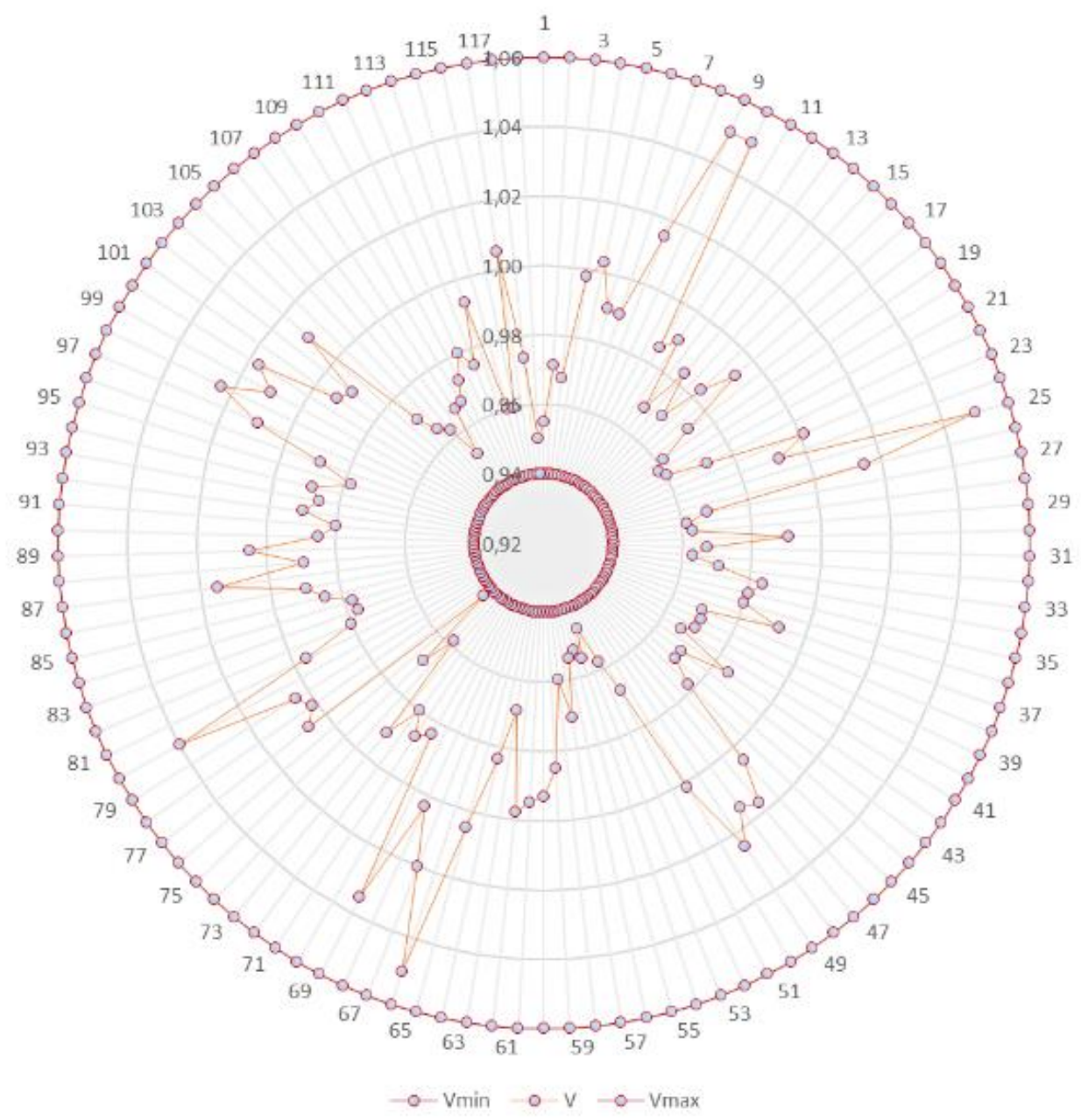

Figure 4. Voltage profile of IEEE 118 bus system at solution point

\section{CONCLUSION}

In this study, the Newton-Raphson method was used to perform load flow analysis and the new program facilitates the data input for the process. On a different sample system, this new data input method can be used to provide data input without running the 
load flow program. This new method makes it easier to correctly input data for the solutions to high-dimensional systems. It also prevents erroneous data input. The data input innovation developed in this study can also be improved for future studies and used for economic power distribution and short term hydrothermal coordination problems.

\section{ACKNOWLEDGMENT}

This work was supported by the Dumlupinar University Scientific Research Projects Commission under the 2016-65 project.

\section{REFERENCES}

Allan, R.N. and Al-Shakarchi, M.R.G. "Probabilistic techniques in a.c. load-flow analysis", Proceedings of the Institution of Electrical Engineers, vol. 124, no. 2, pp. 154-160, February 1977.

Dagur, D., Parimi, M. and Wagh, S.R., "Prediction of cascade failure using probabilistic approach with AC load flow" IEEE Innovative Smart Grid Technologies-Asia, 2014, pp. 542-547.

Kothari, D.P. and Dhillon, J.S., Power System Optimization, PHI, New Delhi, 2007.

Özyön S., The application of genetic algorithm to some environmental economic power dispatch problems, Msc. Thesis, Dumlupınar University, Kütahya, 2009.

Panosyan, A. and Oswald, B.R., "Modified Newton-Raphson load flow analysis for integrated AC/DC power systems" 39th International Universities Power Engineering Conference, 2004, pp. 1223-1227.

Power Systems Test Case Archive. (n.d.). Retrieved September 12, 2017, from https://www2.ee.washington.edu/research/pstca/ MatPower, Retrieved September 12, 2017, from http://www.pserc.cornell.edu/matpower/

Revanthi, A.A., Load flow and optimal power flow analysis using evolutionary computational techniques, Phd Thesis, Anna University, Faculty of Electrical and Electronics Engineering, 2008.

Rossoni, P., Rosa, W.M. and Belati, E.A. "Linearized AC load flow applied to analysis in electric power systems", IEEE Latin America Transactions, vol. 14, no. 9, pp. 4048-4053, September 2016.

Wood, A.J., Wollenberg, B.F. and Sheble, G.B., Power Generation Operation and Control, IEEE \& Wiley, Third Edition, USA, 2013.

Zahlay, F.D., Strategies, Methods and Tools for Solving Long-term Transmission Expansion Planning in Large-scale Power Systems, Ph.D. Thesis, Spain, 2016. 
Appendix

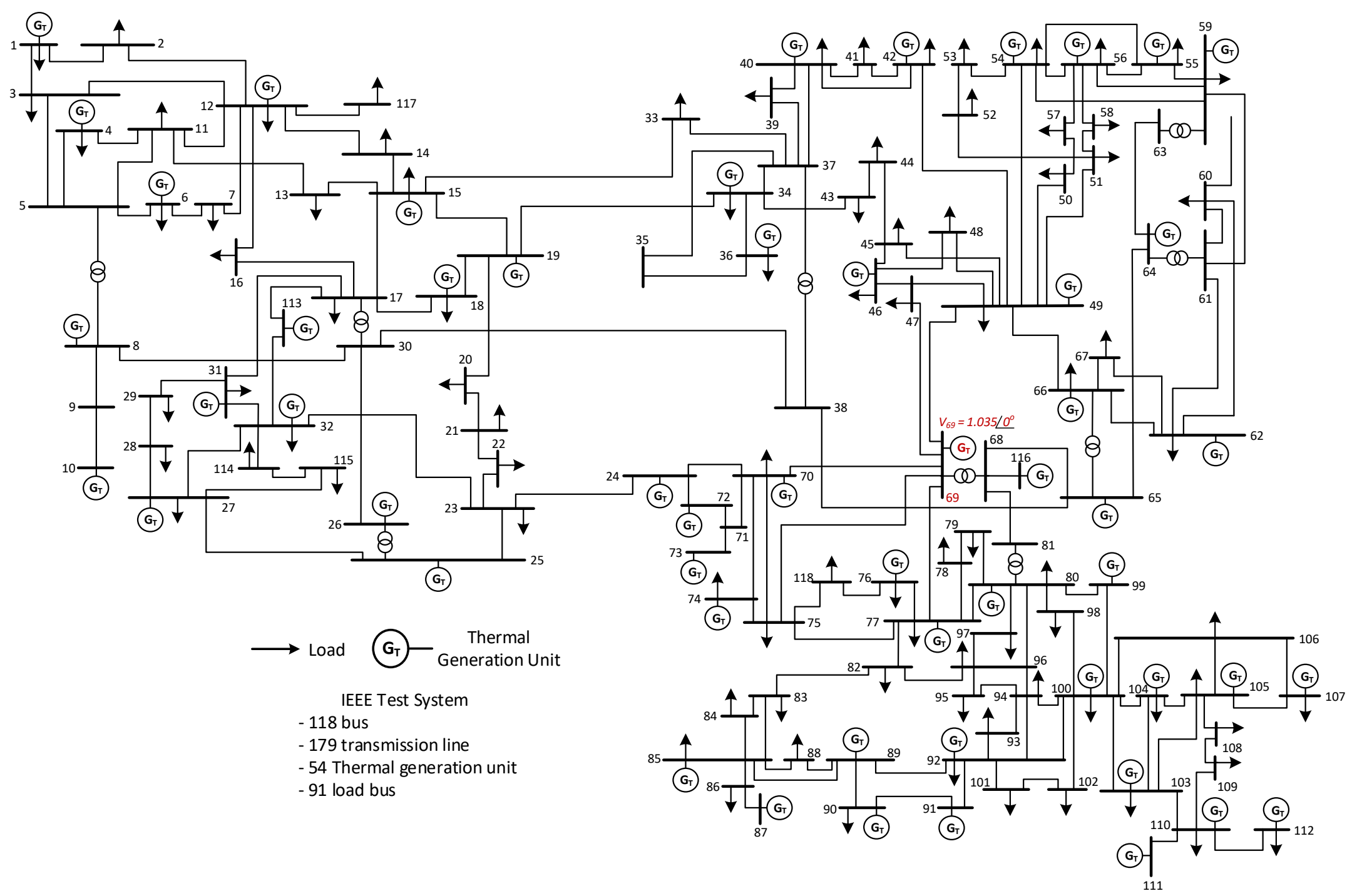

Appendix Figure 1. Single line diagram of the test system. 
Appendix Table 1. Values of nominal $\pi$ equivalent circuits of transmission lines in the sample system

\begin{tabular}{|c|c|c|c|c|c|c|c|}
\hline $\begin{array}{c}\text { Line } \\
\text { no }\end{array}$ & $\begin{array}{c}\text { From } \\
\text { bus }\end{array}$ & To bus & $R(p u)$ & $X(p u)$ & $B(p u)$ & Tap & $S_{L}{ }^{\max }$ \\
\hline 1 & 1 & 2 & 0,03030 & 0,09990 & 0,02540 & - & 1,750 \\
\hline 2 & 1 & 3 & 0,01290 & 0,04240 & 0,01082 & - & 1,750 \\
\hline 3 & 4 & 5 & 0,00176 & 0,00798 & 0,00210 & - & 5,000 \\
\hline 4 & 3 & 5 & 0,02410 & 0,10800 & 0,02840 & - & 1,750 \\
\hline 5 & 5 & 6 & 0,01190 & 0,05400 & 0,01426 & - & 1,750 \\
\hline 6 & 6 & 7 & 0,00459 & 0,02080 & 0,00550 & - & 1,750 \\
\hline 7 & 8 & 9 & 0,00244 & 0,03050 & 1,16200 & - & 5,000 \\
\hline 8 & 8 & 5 & - & 0,02670 & - & 0,985 & 5,000 \\
\hline 9 & 9 & 10 & 0,00258 & 0,03220 & 1,23000 & - & 5,000 \\
\hline 10 & 4 & 11 & 0,02090 & 0,06880 & 0,01748 & - & 1,750 \\
\hline 11 & 5 & 11 & 0,02030 & 0,06820 & 0,01738 & - & 1,750 \\
\hline 12 & 11 & 12 & 0,00595 & 0,01960 & 0,00502 & - & 1,750 \\
\hline 13 & 2 & 12 & 0,01870 & 0,06160 & 0,01572 & - & 1,750 \\
\hline 14 & 3 & 12 & 0,04840 & 0,16000 & 0,04060 & - & 1,750 \\
\hline 15 & 7 & 12 & 0,00862 & 0,03400 & 0,00874 & - & 1,750 \\
\hline 16 & 11 & 13 & 0,02225 & 0,07310 & 0,01876 & - & 1,750 \\
\hline 17 & 12 & 14 & 0,02150 & 0,07070 & 0,01816 & - & 1,750 \\
\hline 18 & 13 & 15 & 0,07440 & 0,24440 & 0,06268 & - & 1,750 \\
\hline 19 & 14 & 15 & 0,05950 & 0,19500 & 0,05020 & - & 1,750 \\
\hline 20 & 12 & 16 & 0,02120 & 0,08340 & 0,02140 & - & 1,750 \\
\hline 21 & 15 & 17 & 0,01320 & 0,04370 & 0,04440 & - & 5,000 \\
\hline 22 & 16 & 17 & 0,04540 & 0,18010 & 0,04660 & - & 1,750 \\
\hline 23 & 17 & 18 & 0,01230 & 0,05050 & 0,01298 & - & 1,750 \\
\hline 24 & 18 & 19 & 0,01119 & 0,04930 & 0,01142 & - & 1,750 \\
\hline 25 & 19 & 20 & 0,02520 & 0,11700 & 0,02980 & - & 1,750 \\
\hline 26 & 15 & 19 & 0,01200 & 0,03940 & 0,01010 & - & 1,750 \\
\hline 27 & 20 & 21 & 0,01830 & 0,08490 & 0,02160 & - & 1,750 \\
\hline 28 & 21 & 22 & 0,02090 & 0,09700 & 0,02460 & - & 1,750 \\
\hline 29 & 22 & 23 & 0,03420 & 0,15900 & 0,04040 & - & 1,750 \\
\hline 30 & 23 & 24 & 0,01350 & 0,04920 & 0,04980 & - & 1,750 \\
\hline 31 & 23 & 25 & 0,01560 & 0,08000 & 0,08640 & - & 5,000 \\
\hline 32 & 26 & 25 & - & 0,03820 & - & 0,960 & 5,000 \\
\hline 33 & 25 & 27 & 0,03180 & 0,16300 & 0,17640 & - & 5,000 \\
\hline 34 & 27 & 28 & 0,01913 & 0,08550 & 0,02160 & - & 1,750 \\
\hline 35 & 28 & 29 & 0,02370 & 0,09430 & 0,02380 & - & 1,750 \\
\hline 36 & 30 & 17 & - & 0,03880 & - & 0,960 & 5,000 \\
\hline 37 & 8 & 30 & 0,00431 & 0,05040 & 0,51400 & - & 1,750 \\
\hline 38 & 26 & 30 & 0,00799 & 0,08600 & 0,90800 & - & 5,000 \\
\hline 39 & 17 & 31 & 0,04740 & 0,15630 & 0,03990 & - & 1,750 \\
\hline 40 & 29 & 31 & 0,01080 & 0,03310 & 0,00830 & - & 1,750 \\
\hline 41 & 23 & 32 & 0,03170 & 0,11530 & 0,11730 & - & 1,40 \\
\hline 42 & 31 & 32 & 0,02980 & 0,09850 & 0,02510 & - & 1,750 \\
\hline 43 & 27 & 32 & 0,02290 & 0,07550 & 0,01926 & - & 1,750 \\
\hline 44 & 15 & 33 & 0,03800 & 0,12440 & 0,03194 & - & 1,750 \\
\hline 45 & 19 & 34 & 0,07520 & 0,24700 & 0,06320 & - & 1,750 \\
\hline 46 & 35 & 36 & 0,00224 & 0,01020 & 0,00268 & - & 1,750 \\
\hline 47 & 35 & 37 & 0,01100 & 0,04970 & 0,01318 & - & 1,750 \\
\hline 48 & 33 & 37 & 0,04150 & 0,14200 & 0,03660 & - & 1,750 \\
\hline 49 & 34 & 36 & 0,00871 & 0,02680 & 0,00568 & - & 1,750 \\
\hline 50 & 34 & 37 & 0,00256 & 0,00940 & 0,00984 & - & 5,000 \\
\hline 51 & 38 & 37 & - & 0,03750 & - & 0,935 & 5,000 \\
\hline 52 & 37 & 39 & 0,03210 & 0,10600 & 0,02700 & - & 1,750 \\
\hline
\end{tabular}




\begin{tabular}{|c|c|c|c|c|c|c|c|}
\hline 53 & 37 & 40 & 0,05930 & 0,16800 & 0,04200 & - & 1,750 \\
\hline 54 & 30 & 38 & 0,00464 & 0,05400 & 0,42200 & - & 1,750 \\
\hline 55 & 39 & 40 & 0,01840 & 0,06050 & 0,01552 & - & 1,750 \\
\hline 56 & 40 & 41 & 0,01450 & 0,04870 & 0,01222 & - & 1,750 \\
\hline 57 & 40 & 42 & 0,05550 & 0,18300 & 0,04660 & - & 1,750 \\
\hline 58 & 41 & 42 & 0,04100 & 0,13500 & 0,03440 & - & 1,750 \\
\hline 59 & 43 & 44 & 0,06080 & 0,24540 & 0,06068 & - & 1,750 \\
\hline 60 & 34 & 43 & 0,04130 & 0,16810 & 0,04226 & - & 1,750 \\
\hline 61 & 44 & 45 & 0,02240 & 0,09010 & 0,02240 & - & 1,750 \\
\hline 62 & 45 & 46 & 0,04000 & 0,13560 & 0,03320 & - & 1,750 \\
\hline 63 & 46 & 47 & 0,03800 & 0,12700 & 0,03160 & - & 1,750 \\
\hline 64 & 46 & 48 & 0,06010 & 0,18900 & 0,04720 & - & 1,750 \\
\hline 65 & 47 & 49 & 0,01910 & 0,06250 & 0,01604 & - & 1,750 \\
\hline 66 & 42 & 49 & 0,03575 & 0,16150 & 0,17200 & - & 2,300 \\
\hline 67 & 45 & 49 & 0,06840 & 0,18600 & 0,04440 & - & 1,750 \\
\hline 68 & 48 & 49 & 0,01790 & 0,05050 & 0,01258 & - & 1,750 \\
\hline 69 & 49 & 50 & 0,02670 & 0,07520 & 0,01874 & - & 1,750 \\
\hline 70 & 49 & 51 & 0,04860 & 0,13700 & 0,03420 & - & 1,750 \\
\hline 71 & 51 & 52 & 0,02030 & 0,05880 & 0,01396 & - & 1,750 \\
\hline 72 & 52 & 53 & 0,04050 & 0,16350 & 0,04058 & - & 1,750 \\
\hline 73 & 53 & 54 & 0,02630 & 0,12200 & 0,03100 & - & 1,750 \\
\hline 74 & 49 & 54 & 0,03993 & 0,14507 & 0,14680 & - & 2,300 \\
\hline 75 & 54 & 55 & 0,01690 & 0,07070 & 0,02020 & - & 1,750 \\
\hline 76 & 54 & 56 & 0,00275 & 0,00955 & 0,00732 & - & 1,750 \\
\hline 77 & 55 & 56 & 0,00488 & 0,01510 & 0,00374 & - & 1,750 \\
\hline 78 & 56 & 57 & 0,03430 & 0,09660 & 0,02420 & - & 1,750 \\
\hline 79 & 50 & 57 & 0,04740 & 0,13400 & 0,03320 & - & 1,750 \\
\hline 80 & 56 & 58 & 0,03430 & 0,09660 & 0,02420 & - & 1,750 \\
\hline 81 & 51 & 58 & 0,02550 & 0,07190 & 0,01788 & - & 1,750 \\
\hline 82 & 54 & 59 & 0,05030 & 0,22930 & 0,05980 & - & 1,750 \\
\hline 83 & 56 & 59 & 0,04070 & 0,12243 & 0,11050 & - & 2,300 \\
\hline 84 & 55 & 59 & 0,04739 & 0,21580 & 0,05646 & - & 1,750 \\
\hline 85 & 59 & 60 & 0,03170 & 0,14500 & 0,03760 & - & 1,750 \\
\hline 86 & 59 & 61 & 0,03280 & 0,15000 & 0,03880 & - & 1,750 \\
\hline 87 & 60 & 61 & 0,00264 & 0,01350 & 0,01456 & - & 5,000 \\
\hline 88 & 60 & 62 & 0,01230 & 0,05610 & 0,01468 & - & 1,750 \\
\hline 89 & 61 & 62 & 0,00824 & 0,03760 & 0,00980 & - & 1,750 \\
\hline 90 & 63 & 59 & - & 0,03860 & - & 0,960 & 5,000 \\
\hline 91 & 63 & 64 & 0,00172 & 0,02000 & 0,21600 & - & 5,000 \\
\hline 92 & 64 & 61 & - & 0,02680 & - & 0,985 & 5,000 \\
\hline 93 & 38 & 65 & 0,00901 & 0,09860 & 1,04600 & - & 5,000 \\
\hline 94 & 64 & 65 & 0,00269 & 0,03020 & 0,38000 & - & 5,000 \\
\hline 95 & 49 & 66 & 0,00900 & 0,04595 & 0,04960 & - & 8,000 \\
\hline 96 & 62 & 66 & 0,04820 & 0,21800 & 0,05780 & - & 1,750 \\
\hline 97 & 62 & 67 & 0,02580 & 0,11700 & 0,03100 & - & 1,750 \\
\hline 98 & 65 & 66 & - & 0,03700 & - & 0,935 & 5,000 \\
\hline 99 & 66 & 67 & 0,02240 & 0,10150 & 0,02682 & - & 1,750 \\
\hline 100 & 65 & 68 & 0,00138 & 0,01600 & 0,63800 & - & 5,000 \\
\hline 101 & 47 & 69 & 0,08440 & 0,27780 & 0,07092 & - & 1,750 \\
\hline 102 & 49 & 69 & 0,09850 & 0,32400 & 0,08280 & - & 1,750 \\
\hline 103 & 68 & 69 & - & 0,03700 & - & 0,935 & 5,000 \\
\hline 104 & 69 & 70 & 0,03000 & 0,12700 & 0,12200 & - & 5,000 \\
\hline 105 & 24 & 70 & 0,00221 & 0,41150 & 0,10198 & - & 1,750 \\
\hline 106 & 70 & 71 & 0,00882 & 0,03550 & 0,00878 & - & 1,750 \\
\hline 107 & 24 & 72 & 0,04880 & 0,19600 & 0,04880 & - & 1,750 \\
\hline
\end{tabular}




\begin{tabular}{|c|c|c|c|c|c|c|c|}
\hline 108 & 71 & 72 & 0,04460 & 0,18000 & 0,04444 & - & 1,750 \\
\hline 109 & 71 & 73 & 0,00866 & 0,04540 & 0,01178 & - & 1,750 \\
\hline 110 & 70 & 74 & 0,04010 & 0,13230 & 0,03368 & - & 1,750 \\
\hline 111 & 70 & 75 & 0,04280 & 0,14100 & 0,03600 & - & 1,750 \\
\hline 112 & 69 & 75 & 0,04050 & 0,12200 & 0,12400 & - & 5,000 \\
\hline 113 & 74 & 75 & 0,01230 & 0,04060 & 0,01034 & - & 1,750 \\
\hline 114 & 76 & 77 & 0,04440 & 0,14800 & 0,03680 & - & 1,750 \\
\hline 115 & 69 & 77 & 0,03090 & 0,10100 & 0,10380 & - & 1,750 \\
\hline 116 & 75 & 77 & 0,06010 & 0,19990 & 0,04978 & - & 1,750 \\
\hline 117 & 77 & 78 & 0,00376 & 0,01240 & 0,01264 & - & 1,750 \\
\hline 118 & 78 & 79 & 0,00546 & 0,02440 & 0,00648 & - & 1,750 \\
\hline 119 & 77 & 80 & 0,01088 & 0,03321 & 0,07000 & - & 8,000 \\
\hline 120 & 79 & 80 & 0,01560 & 0,07040 & 0,01870 & - & 1,750 \\
\hline 121 & 68 & 81 & 0,00175 & 0,02020 & 0,80800 & - & 5,000 \\
\hline 122 & 81 & 80 & - & 0,03700 & - & 0,935 & 5,000 \\
\hline 123 & 77 & 82 & 0,02980 & 0,08530 & 0,08174 & - & 2,000 \\
\hline 124 & 82 & 83 & 0,01120 & 0,03665 & 0,03796 & - & 2,000 \\
\hline 125 & 83 & 84 & 0,06250 & 0,13200 & 0,02580 & - & 1,750 \\
\hline 126 & 83 & 85 & 0,04300 & 0,14800 & 0,03480 & - & 1,750 \\
\hline 127 & 84 & 85 & 0,03020 & 0,06410 & 0,01234 & - & 1,750 \\
\hline 128 & 85 & 86 & 0,03500 & 0,12300 & 0,02760 & - & 5,000 \\
\hline 129 & 86 & 87 & 0,02828 & 0,20740 & 0,04450 & - & 5,000 \\
\hline 130 & 85 & 88 & 0,02000 & 0,10200 & 0,02760 & - & 1,750 \\
\hline 131 & 85 & 89 & 0,02390 & 0,17300 & 0,04700 & - & 1,750 \\
\hline 132 & 88 & 89 & 0,01390 & 0,07120 & 0,01934 & - & 5,000 \\
\hline 133 & 89 & 90 & 0,01638 & 0,06517 & 0,15880 & - & 8,000 \\
\hline 134 & 90 & 91 & 0,02540 & 0,08360 & 0,02140 & - & 1,750 \\
\hline 135 & 89 & 92 & 0,00799 & 0,03829 & 0,09620 & - & 8,000 \\
\hline 136 & 91 & 92 & 0,03870 & 0,12720 & 0,03268 & - & 1,750 \\
\hline 137 & 92 & 93 & 0,02580 & 0,08480 & 0,02180 & - & 1,750 \\
\hline 138 & 92 & 94 & 0,04810 & 0,15800 & 0,04060 & - & 1,750 \\
\hline 139 & 93 & 94 & 0,02230 & 0,07320 & 0,01876 & - & 1,750 \\
\hline 140 & 94 & 95 & 0,01320 & 0,04340 & 0,01110 & - & 1,750 \\
\hline 141 & 80 & 96 & 0,03560 & 0,18200 & 0,04940 & - & 1,750 \\
\hline 142 & 82 & 96 & 0,01620 & 0,05300 & 0,05440 & - & 1,750 \\
\hline 143 & 94 & 96 & 0,02690 & 0,08690 & 0,02300 & - & 1,750 \\
\hline 144 & 80 & 97 & 0,01830 & 0,09340 & 0,02540 & - & 1,750 \\
\hline 145 & 80 & 98 & 0,02380 & 0,10800 & 0,02860 & - & 1,750 \\
\hline 146 & 80 & 99 & 0,04540 & 0,20600 & 0,05460 & - & 2,000 \\
\hline 147 & 92 & 100 & 0,06480 & 0,29500 & 0,04720 & - & 1,750 \\
\hline 148 & 94 & 100 & 0,01780 & 0,05800 & 0,06040 & - & 1,750 \\
\hline 149 & 95 & 96 & 0,01710 & 0,05470 & 0,01474 & - & 1,750 \\
\hline 150 & 96 & 97 & 0,01730 & 0,08850 & 0,02400 & - & 1,750 \\
\hline 151 & 98 & 100 & 0,03970 & 0,17900 & 0,04760 & - & 1,750 \\
\hline 152 & 99 & 100 & 0,01800 & 0,08130 & 0,02160 & - & 1,750 \\
\hline 153 & 100 & 101 & 0,02770 & 0,12620 & 0,03280 & - & 1,750 \\
\hline 154 & 92 & 102 & 0,01230 & 0,05590 & 0,01464 & - & 1,750 \\
\hline 155 & 101 & 102 & 0,02460 & 0,11200 & 0,02940 & - & 1,750 \\
\hline 156 & 100 & 103 & 0,01600 & 0,05250 & 0,05360 & - & 5,000 \\
\hline 157 & 100 & 104 & 0,04510 & 0,20400 & 0,05410 & - & 1,750 \\
\hline 158 & 103 & 104 & 0,04660 & 0,15840 & 0,04070 & - & 1,750 \\
\hline 159 & 103 & 105 & 0,05350 & 0,16250 & 0,04080 & - & 1,750 \\
\hline 160 & 100 & 106 & 0,06050 & 0,22900 & 0,06200 & - & 1,750 \\
\hline 161 & 104 & 105 & 0,00994 & 0,03780 & 0,00986 & - & 1,750 \\
\hline 162 & 105 & 106 & 0,01400 & 0,05470 & 0,01434 & - & 1,750 \\
\hline
\end{tabular}




\begin{tabular}{|l|c|c|c|c|c|c|c|}
\hline $\mathbf{1 6 3}$ & 105 & 107 & 0,05300 & 0,18300 & 0,04720 & - & 1,750 \\
\hline $\mathbf{1 6 4}$ & 105 & 108 & 0,02610 & 0,07030 & 0,01844 & - & 1,750 \\
\hline $\mathbf{1 6 5}$ & 106 & 107 & 0,05300 & 0,18300 & 0,04720 & - & 1,750 \\
\hline $\mathbf{1 6 6}$ & 108 & 109 & 0,01050 & 0,02880 & 0,00760 & - & 1,750 \\
\hline $\mathbf{1 6 7}$ & 103 & 110 & 0,03906 & 0,18130 & 0,04610 & - & 1,750 \\
\hline $\mathbf{1 6 8}$ & 109 & 110 & 0,02780 & 0,07620 & 0,02020 & - & 1,750 \\
\hline $\mathbf{1 6 9}$ & 110 & 111 & 0,02200 & 0,07550 & 0,02000 & - & 1,750 \\
\hline $\mathbf{1 7 0}$ & 110 & 112 & 0,02470 & 0,06400 & 0,06200 & - & 1,750 \\
\hline $\mathbf{1 7 1}$ & 17 & 113 & 0,00913 & 0,03010 & 0,00768 & - & 1,750 \\
\hline $\mathbf{1 7 2}$ & 32 & 113 & 0,06150 & 0,20300 & 0,05180 & - & 5,000 \\
\hline $\mathbf{1 7 3}$ & 32 & 114 & 0,01350 & 0,06120 & 0,01628 & - & 1,750 \\
\hline $\mathbf{1 7 4}$ & 27 & 115 & 0,01640 & 0,07410 & 0,01972 & - & 1,750 \\
\hline $\mathbf{1 7 5}$ & 114 & 115 & 0,00230 & 0,01040 & 0,00276 & - & 1,750 \\
\hline $\mathbf{1 7 6}$ & 68 & 116 & 0,00034 & 0,00405 & 0,16400 & - & 5,000 \\
\hline $\mathbf{1 7 7}$ & 12 & 117 & 0,03290 & 0,14000 & 0,03580 & - & 1,750 \\
\hline $\mathbf{1 7 8}$ & 75 & 118 & 0,01450 & 0,04810 & 0,01198 & - & 1,750 \\
\hline $\mathbf{1 7 9}$ & 76 & 118 & 0,01640 & 0,05440 & 0,01356 & - & 1,750 \\
\hline
\end{tabular}

Appendix Table 2. Active and reactive load values in the sample system.

\begin{tabular}{|c|c|c|c|c|c|}
\hline $\begin{array}{c}\text { Bus } \\
\text { No }\end{array}$ & $\boldsymbol{P}_{\text {load }}(\boldsymbol{p u})$ & $\boldsymbol{Q}_{\text {load }}(\boldsymbol{p u})$ & $\begin{array}{c}\text { Bus } \\
\mathbf{N o}\end{array}$ & $\boldsymbol{P}_{\text {load }}(\boldsymbol{p u})$ & $\boldsymbol{Q}_{\text {load }}(\boldsymbol{p u})$ \\
\hline $\mathbf{1}$ & 0,5100 & 0,2700 & $\mathbf{6 0}$ & 0,7800 & 0,0300 \\
\hline $\mathbf{2}$ & 0,2000 & 0,0900 & $\mathbf{6 1}$ & - & - \\
\hline $\mathbf{3}$ & 0,3900 & 0,1000 & $\mathbf{6 2}$ & 0,7700 & 0,1400 \\
\hline $\mathbf{4}$ & 0,3900 & 0,1200 & $\mathbf{6 3}$ & - & - \\
\hline $\mathbf{5}$ & - & - & $\mathbf{6 4}$ & - & - \\
\hline $\mathbf{6}$ & 0,5200 & 0,2200 & $\mathbf{6 5}$ & - & - \\
\hline $\mathbf{7}$ & 0,1900 & 0,0200 & $\mathbf{6 6}$ & 0,3900 & 0,1800 \\
\hline $\mathbf{8}$ & 0,2800 & 0,0000 & $\mathbf{6 7}$ & 0,2800 & 0,0700 \\
\hline $\mathbf{9}$ & - & - & $\mathbf{6 8}$ & - & - \\
\hline $\mathbf{1 0}$ & - & - & $\mathbf{6 9}$ & - & - \\
\hline $\mathbf{1 1}$ & 0,7000 & 0,2300 & $\mathbf{7 0}$ & 0,6600 & 0,2000 \\
\hline $\mathbf{1 2}$ & 0,4700 & 0,1000 & $\mathbf{7 1}$ & - & - \\
\hline $\mathbf{1 3}$ & 0,3400 & 0,1600 & $\mathbf{7 2}$ & 0,1200 & - \\
\hline $\mathbf{1 4}$ & 0,1400 & 0,0100 & $\mathbf{7 3}$ & 0,0600 & - \\
\hline $\mathbf{1 5}$ & 0,9000 & 0,3000 & $\mathbf{7 4}$ & 0,6800 & 0,2700 \\
\hline $\mathbf{1 6}$ & 0,2500 & 0,1000 & $\mathbf{7 5}$ & 0,4700 & 0,1100 \\
\hline $\mathbf{1 7}$ & 0,1100 & 0,0300 & $\mathbf{7 6}$ & 0,6800 & 0,3600 \\
\hline $\mathbf{1 8}$ & 0,6000 & 0,3400 & $\mathbf{7 7}$ & 0,6100 & 0,2800 \\
\hline $\mathbf{1 9}$ & 0,4500 & 0,2500 & $\mathbf{7 8}$ & 0,7100 & 0,2600 \\
\hline $\mathbf{2 0}$ & 0,1800 & 0,0300 & $\mathbf{7 9}$ & 0,3900 & 0,3200 \\
\hline $\mathbf{2 1}$ & 0,1400 & 0,0800 & $\mathbf{8 0}$ & 1,3000 & 0,2600 \\
\hline $\mathbf{2 2}$ & 0,1000 & 0,0500 & $\mathbf{8 1}$ & - & - \\
\hline $\mathbf{2 3}$ & 0,0700 & 0,0300 & $\mathbf{8 2}$ & 0,5400 & 0,2700 \\
\hline $\mathbf{2 4}$ & 0,1300 & - & $\mathbf{8 3}$ & 0,2000 & 0,1000 \\
\hline $\mathbf{2 5}$ & - & - & $\mathbf{8 4}$ & 0,1100 & 0,0700 \\
\hline $\mathbf{2 6}$ & - & - & $\mathbf{8 5}$ & 0,2400 & 0,1500 \\
\hline $\mathbf{2 7}$ & 0,7100 & 0,1300 & $\mathbf{8 6}$ & 0,2100 & 0,1000 \\
\hline $\mathbf{2 8}$ & 0,1700 & 0,0700 & $\mathbf{8 7}$ & - & - \\
\hline $\mathbf{2 9}$ & 0,2400 & 0,0400 & $\mathbf{8 8}$ & 0,4800 & 0,1000 \\
\hline $\mathbf{3 0}$ & - & - & $\mathbf{8 9}$ & - & - \\
\hline $\mathbf{3 1}$ & 0,4300 & 0,2700 & $\mathbf{9 0}$ & 1,6300 & 0,4200 \\
\hline $\mathbf{3 2}$ & 0,5900 & 0,2300 & $\mathbf{9 1}$ & 0,1000 & - \\
\hline $\mathbf{3 3}$ & 0,2300 & 0,0900 & $\mathbf{9 2}$ & 0,6500 & 0,1000 \\
\hline $\mathbf{3 4}$ & 0,5900 & 0,2600 & $\mathbf{9 3}$ & 0,1200 & 0,0700 \\
\hline & & & & & \\
\hline
\end{tabular}




\begin{tabular}{|c|c|c|c|c|c|}
\hline $\mathbf{3 5}$ & 0,3300 & 0,0900 & $\mathbf{9 4}$ & 0,3000 & 0,1600 \\
\hline $\mathbf{3 6}$ & 0,3100 & 0,1700 & $\mathbf{9 5}$ & 0,4200 & 0,3100 \\
\hline $\mathbf{3 7}$ & - & - & $\mathbf{9 6}$ & 0,3800 & 0,1500 \\
\hline $\mathbf{3 8}$ & - & - & $\mathbf{9 7}$ & 0,1500 & 0,0900 \\
\hline $\mathbf{3 9}$ & 0,2700 & 0,1100 & $\mathbf{9 8}$ & 0,3400 & 0,0800 \\
\hline $\mathbf{4 0}$ & 0,6600 & 0,2300 & $\mathbf{9 9}$ & 0,4200 & - \\
\hline $\mathbf{4 1}$ & 0,3700 & 0,1000 & $\mathbf{1 0 0}$ & 0,3700 & 0,1800 \\
\hline $\mathbf{4 2}$ & 0,9600 & 0,2300 & $\mathbf{1 0 1}$ & 0,2200 & 0,1500 \\
\hline $\mathbf{4 3}$ & 0,1800 & 0,0700 & $\mathbf{1 0 2}$ & 0,0500 & 0,0300 \\
\hline $\mathbf{4 4}$ & 0,1600 & 0,0800 & $\mathbf{1 0 3}$ & 0,2300 & 0,1600 \\
\hline $\mathbf{4 5}$ & 0,5300 & 0,2200 & $\mathbf{1 0 4}$ & 0,3800 & 0,2500 \\
\hline $\mathbf{4 6}$ & 0,2800 & 0,1000 & $\mathbf{1 0 5}$ & 0,3100 & 0,2600 \\
\hline $\mathbf{4 7}$ & 0,3400 & - & $\mathbf{1 0 6}$ & 0,4300 & 0,1600 \\
\hline $\mathbf{4 8}$ & 0,2000 & 0,1100 & $\mathbf{1 0 7}$ & 0,5000 & 0,1200 \\
\hline $\mathbf{4 9}$ & 0,8700 & 0,3000 & $\mathbf{1 0 8}$ & 0,0200 & 0,0100 \\
\hline $\mathbf{5 0}$ & 0,1700 & 0,0400 & $\mathbf{1 0 9}$ & 0,0800 & 0,0300 \\
\hline $\mathbf{5 1}$ & 0,1700 & 0,0800 & $\mathbf{1 1 0}$ & 0,3900 & 0,3000 \\
\hline $\mathbf{5 2}$ & 0,1800 & 0,0500 & $\mathbf{1 1 1}$ & - & - \\
\hline $\mathbf{5 3}$ & 0,2300 & 0,1100 & $\mathbf{1 1 2}$ & 0,6800 & 0,1300 \\
\hline $\mathbf{5 4}$ & 1,1300 & 0,3200 & $\mathbf{1 1 3}$ & 0,0600 & - \\
\hline $\mathbf{5 5}$ & 0,6300 & 0,2200 & $\mathbf{1 1 4}$ & 0,0800 & 0,0300 \\
\hline $\mathbf{5 6}$ & 0,8400 & 0,1800 & $\mathbf{1 1 5}$ & 0,2200 & 0,0700 \\
\hline $\mathbf{5 7}$ & 0,1200 & 0,0300 & $\mathbf{1 1 6}$ & 1,8400 & - \\
\hline $\mathbf{5 8}$ & 0,1200 & 0,0300 & $\mathbf{1 1 7}$ & 0,2000 & 0,0800 \\
\hline $\mathbf{5 9}$ & 2,7700 & 1,1300 & $\mathbf{1 1 8}$ & 0,3300 & 0,1500 \\
\hline $\boldsymbol{T}$ Total Load & $\boldsymbol{P}_{\text {load }}: 42,4200$ & $\boldsymbol{Q}_{\text {load }}: 14,3800$ \\
\hline & & & & & \\
\hline
\end{tabular}

Appendix Table 3. AC load flow initial values of the generation units in the sample system

\begin{tabular}{|c|c|c|c|c|c|c|c|c|c|}
\hline $\begin{array}{c}\text { Gen. } \\
\text { Unit }\end{array}$ & $\boldsymbol{B u s}$ & $\begin{array}{c}\boldsymbol{P}_{\boldsymbol{i}} \\
(\mathbf{p u})\end{array}$ & $\begin{array}{c}\boldsymbol{Q}_{\boldsymbol{i}} \\
(\mathbf{p u})\end{array}$ & $\boldsymbol{V}(\mathbf{p u})$ & $\begin{array}{c}\text { Gen. } \\
\text { Unit }\end{array}$ & $\boldsymbol{B u s}$ & $\begin{array}{c}\boldsymbol{P}_{\boldsymbol{i}} \\
(\mathbf{p u})\end{array}$ & $\begin{array}{c}\boldsymbol{Q}_{\boldsymbol{i}} \\
(\mathbf{p u})\end{array}$ & $\boldsymbol{V}(\mathbf{p u})$ \\
\hline $\mathbf{1}$ & 1 & 0,500 & 0,075 & 0,995 & $\mathbf{2 8}$ & 65 & 2,000 & 1,000 & 1,005 \\
\hline $\mathbf{2}$ & 4 & 0,500 & 1,500 & 0,998 & $\mathbf{2 9}$ & 66 & 2,000 & 1,000 & 1,050 \\
\hline $\mathbf{3}$ & 6 & 0,500 & 0,250 & 0,990 & $\mathbf{3 0}$ & 69 & - & - & 1,035 \\
\hline $\mathbf{4}$ & 8 & 0,500 & 1,500 & 1,015 & $\mathbf{3 1}$ & 70 & 0,500 & 0,150 & 0,984 \\
\hline $\mathbf{5}$ & 10 & 2,000 & 1,000 & 1,050 & $\mathbf{3 2}$ & 72 & 0,500 & 0,500 & 0,980 \\
\hline $\mathbf{6}$ & 12 & 1,000 & 0,600 & 0,990 & $\mathbf{3 3}$ & 73 & 0,500 & 0,500 & 0,991 \\
\hline $\mathbf{7}$ & 15 & 0,500 & 0,150 & 0,970 & $\mathbf{3 4}$ & 74 & 0,500 & 0,045 & 0,958 \\
\hline $\mathbf{8}$ & 18 & 0,500 & 0,250 & 0,973 & $\mathbf{3 5}$ & 76 & 0,500 & 0,120 & 0,943 \\
\hline $\mathbf{9}$ & 19 & 0,500 & 0,120 & 0,962 & $\mathbf{3 6}$ & 77 & 0,500 & 0,350 & 1,006 \\
\hline $\mathbf{1 0}$ & 24 & 0,500 & 1,500 & 0,992 & $\mathbf{3 7}$ & 80 & 2,000 & 1,400 & 1,040 \\
\hline $\mathbf{1 1}$ & 25 & 1,000 & 0,700 & 1,050 & $\mathbf{3 8}$ & 85 & 0,500 & 0,120 & 0,985 \\
\hline $\mathbf{1 2}$ & 26 & 2,000 & 5,000 & 1,015 & $\mathbf{3 9}$ & 87 & 0,500 & 5,000 & 1,015 \\
\hline $\mathbf{1 3}$ & 27 & 0,500 & 1,500 & 0,968 & $\mathbf{4 0}$ & 89 & 2,000 & 1,500 & 1,005 \\
\hline $\mathbf{1 4}$ & 31 & 0,500 & 1,500 & 0,967 & $\mathbf{4 1}$ & 90 & 0,500 & 1500 & 0,985 \\
\hline $\mathbf{1 5}$ & 32 & 0,500 & 0,210 & 0,963 & $\mathbf{4 2}$ & 91 & 0,500 & 0,500 & 0,980 \\
\hline $\mathbf{1 6}$ & 34 & 0,500 & 0,120 & 0,984 & $\mathbf{4 3}$ & 92 & 0,500 & 0,045 & 0,990 \\
\hline $\mathbf{1 7}$ & 36 & 0,500 & 0,120 & 0,980 & $\mathbf{4 4}$ & 99 & 0,500 & 0,500 & 1,010 \\
\hline $\mathbf{1 8}$ & 40 & 0,500 & 1,500 & 0,970 & $\mathbf{4 5}$ & 100 & 1,000 & 0,750 & 1,017 \\
\hline $\mathbf{1 9}$ & 42 & 0,500 & 1,500 & 0,985 & $\mathbf{4 6}$ & 103 & 0,500 & 0,200 & 1,010 \\
\hline $\mathbf{2 0}$ & 46 & 0,500 & 0,500 & 1,005 & $\mathbf{4 7}$ & 104 & 0,500 & 0,120 & 0,971 \\
\hline $\mathbf{2 1}$ & 49 & 1,000 & 1,000 & 1,025 & $\mathbf{4 8}$ & 105 & 0,500 & 0,120 & 0,965 \\
\hline $\mathbf{2 2}$ & 54 & 0,500 & 1,500 & 0,955 & $\mathbf{4 9}$ & 107 & 0,500 & 1,000 & 0,952 \\
\hline $\mathbf{2 3}$ & 55 & 0,500 & 0,120 & 0,952 & $\mathbf{5 0}$ & 110 & 0,500 & 0,120 & 0,973 \\
\hline $\mathbf{2 4}$ & 56 & 0,500 & 0,075 & 0,954 & $\mathbf{5 1}$ & 111 & 0,500 & 5,000 & 0,980 \\
\hline $\mathbf{2 5}$ & 59 & 1,000 & 0,900 & 0,985 & $\mathbf{5 2}$ & 112 & 0,500 & 5,000 & 0,975 \\
\hline
\end{tabular}




\begin{tabular}{|l|l|l|l|l|l|l|l|l|l|}
\hline $\mathbf{2 6}$ & 61 & 1,000 & 1,500 & 0,995 & $\mathbf{5 3}$ & 113 & 0,500 & 1,000 & 0,993 \\
\hline $\mathbf{2 7}$ & 62 & 0,500 & 0,100 & 0,998 & $\mathbf{5 4}$ & 116 & 0,500 & 5,000 & 1,005 \\
\hline
\end{tabular}

Appendix Table 4. Operating limit values of generation units in the sample system.

\begin{tabular}{|c|c|c|c|c|c|c|c|}
\hline $\begin{array}{l}\text { Gen. } \\
\text { Unit }\end{array}$ & Bus & $\begin{array}{l}P_{\min } \\
(p u) \\
\end{array}$ & $\begin{array}{l}\boldsymbol{P}_{\max } \\
(p \boldsymbol{p u})\end{array}$ & $\begin{array}{c}Q_{\min } \\
(p u) \\
\end{array}$ & $\begin{array}{c}Q_{\max } \\
(p u)\end{array}$ & $\begin{array}{l}V_{\min } \\
(p u)\end{array}$ & $\begin{array}{l}V_{\max } \\
(p u)\end{array}$ \\
\hline 1 & 1 & 0,0000 & 1,0000 & $-0,0500$ & 0,1500 & 0,9400 & 1,0600 \\
\hline 2 & 4 & 0,0000 & 1,0000 & $-3,0000$ & 3,0000 & 0,9400 & 1,0600 \\
\hline 3 & 6 & 0,0000 & 1,0000 & $-0,1300$ & 0,5000 & 0,9400 & 1,0600 \\
\hline 4 & 8 & 0,0000 & 1,0000 & $-3,0000$ & 3,0000 & 0,9400 & 1,0600 \\
\hline 5 & 10 & 0,0000 & 5,5000 & $-1,4700$ & 2,0000 & 0,9400 & 1,0600 \\
\hline 6 & 12 & 0,0000 & 1,8500 & $-0,3500$ & 1,2000 & 0,9400 & 1,0600 \\
\hline 7 & 15 & 0,0000 & 1,0000 & $-0,1000$ & 0,3000 & 0,9400 & 1,0600 \\
\hline 8 & 18 & 0,0000 & 1,0000 & $-0,1600$ & 0,5000 & 0,9400 & 1,0600 \\
\hline 9 & 19 & 0,0000 & 1,0000 & $-0,0800$ & 0,2400 & 0,9400 & 1,0600 \\
\hline 10 & 24 & 0,0000 & 1,0000 & $-3,0000$ & 3,0000 & 0,9400 & 1,0600 \\
\hline 11 & 25 & 0,0000 & 3,2000 & $-0,4700$ & 1,4000 & 0,9400 & 1,0600 \\
\hline 12 & 26 & 0,0000 & 4,1400 & $\begin{array}{c}- \\
10,0000 \\
\end{array}$ & 10,0000 & 0,9400 & 1,0600 \\
\hline 13 & 27 & 0,0000 & 1,0000 & $-3,0000$ & 3,0000 & 0,9400 & 1,0600 \\
\hline 14 & 31 & 0,0000 & 1,0700 & $-3,0000$ & 3,0000 & 0,9400 & 1,0600 \\
\hline 15 & 32 & 0,0000 & 1,0000 & $-0,1400$ & 0,4200 & 0,9400 & 1,0600 \\
\hline 16 & 34 & 0,0000 & 1,0000 & $-0,0800$ & 0,2400 & 0,9400 & 1,0600 \\
\hline 17 & 36 & 0,0000 & 1,0000 & $-0,0800$ & 0,2400 & 0,9400 & 1,0600 \\
\hline 18 & 40 & 0,0000 & 1,0000 & $-3,0000$ & 3,0000 & 0,9400 & 1,0600 \\
\hline 19 & 42 & 0,0000 & 1,0000 & $-3,0000$ & 3,0000 & 0,9400 & 1,0600 \\
\hline 20 & 46 & 0,0000 & 1,1900 & $-1,0000$ & 1,0000 & 0,9400 & 1,0600 \\
\hline 21 & 49 & 0,0000 & 3,0400 & $-0,8500$ & 2,1000 & 0,9400 & 1,0600 \\
\hline 22 & 54 & 0,0000 & 1,4800 & $-3,0000$ & 3,0000 & 0,9400 & 1,0600 \\
\hline 23 & 55 & 0,0000 & 1,0000 & $-0,0800$ & 0,2300 & 0,9400 & 1,0600 \\
\hline 24 & 56 & 0,0000 & 1,0000 & $-0,0800$ & 0,1500 & 0,9400 & 1,0600 \\
\hline 25 & 59 & 0,0000 & 2,5500 & $-0,6000$ & 1,8000 & 0,9400 & 1,0600 \\
\hline 26 & 61 & 0,0000 & 2,6000 & $-1,0000$ & 3,0000 & 0,9400 & 1,0600 \\
\hline 27 & 62 & 0,0000 & 1,0000 & $-0,2000$ & 0,2000 & 0,9400 & 1,0600 \\
\hline 28 & 65 & 0,0000 & 4,9100 & $-0,6700$ & 2,0000 & 0,9400 & 1,0600 \\
\hline 29 & 66 & 0,0000 & 4,9200 & $-0,6700$ & 2,0000 & 0,9400 & 1,0600 \\
\hline 30 & 69 & 0,0000 & 8,0520 & $-3,0000$ & 3,0000 & 0,9400 & 1,0600 \\
\hline 31 & 70 & 0,0000 & 1,0000 & $-0,1000$ & 0,3200 & 0,9400 & 1,0600 \\
\hline 32 & 72 & 0,0000 & 1,0000 & $-1,0000$ & 1,0000 & 0,9400 & 1,0600 \\
\hline 33 & 73 & 0,0000 & 1,0000 & $-1,0000$ & 1,0000 & 0,9400 & 1,0600 \\
\hline 34 & 74 & 0,0000 & 1,0000 & $-0,0600$ & 0,0900 & 0,9400 & 1,0600 \\
\hline 35 & 76 & 0,0000 & 1,0000 & $-0,0800$ & 0,2300 & 0,9400 & 1,0600 \\
\hline 36 & 77 & 0,0000 & 1,0000 & $-0,2000$ & 0,7000 & 0,9400 & 1,0600 \\
\hline 37 & 80 & 0,0000 & 5,7700 & $-1,6500$ & 2,8000 & 0,9400 & 1,0600 \\
\hline 38 & 85 & 0,0000 & 1,0000 & $-0,0800$ & 0,2300 & 0,9400 & 1,0600 \\
\hline 39 & 87 & 0,0000 & 1,0400 & $-1,0000$ & 10,0000 & 0,9400 & 1,0600 \\
\hline 40 & 89 & 0,0000 & 7,0700 & $-2,1000$ & 3,0000 & 0,9400 & 1,0600 \\
\hline 41 & 90 & 0,0000 & 1,0000 & $-3,0000$ & 3,0000 & 0,9400 & 1,0600 \\
\hline 42 & 91 & 0,0000 & 1,0000 & $-1,0000$ & 1,0000 & 0,9400 & 1,0600 \\
\hline 43 & 92 & 0,0000 & 1,0000 & $-0,0300$ & 0,0900 & 0,9400 & 1,0600 \\
\hline 44 & 99 & 0,0000 & 1,0000 & $-1,0000$ & 1,0000 & 0,9400 & 1,0600 \\
\hline 45 & 100 & 0,0000 & 3,5200 & $-0,5000$ & 1,5500 & 0,9400 & 1,0600 \\
\hline 46 & 103 & 0,0000 & 1,4000 & $-0,1500$ & 0,4000 & 0,9400 & 1,0600 \\
\hline
\end{tabular}




\begin{tabular}{|l|c|c|c|c|c|c|c|}
\hline $\mathbf{4 7}$ & 104 & 0,0000 & 1,0000 & $-0,0800$ & 0,2300 & 0,9400 & 1,0600 \\
\hline $\mathbf{4 8}$ & 105 & 0,0000 & 1,0000 & $-0,0800$ & 0,2300 & 0,9400 & 1,0600 \\
\hline $\mathbf{4 9}$ & 107 & 0,0000 & 1,0000 & $-2,0000$ & 2,0000 & 0,9400 & 1,0600 \\
\hline $\mathbf{5 0}$ & 110 & 0,0000 & 1,0000 & $-0,0800$ & 0,2300 & 0,9400 & 1,0600 \\
\hline $\mathbf{5 1}$ & 111 & 0,0000 & 1,3600 & $-1,0000$ & 10,0000 & 0,9400 & 1,0600 \\
\hline $\mathbf{5 2}$ & 112 & 0,0000 & 1,0000 & $-1,0000$ & 10,0000 & 0,9400 & 1,0600 \\
\hline $\mathbf{5 3}$ & 113 & 0,0000 & 1,0000 & $-1,0000$ & 2,0000 & 0,9400 & 1,0600 \\
\hline $\mathbf{5 4}$ & 116 & 0,0000 & 1,0000 & - & 10,0000 & 0,9400 & 1,0600 \\
\hline
\end{tabular}

Appendix Table 5. The values obtained as a result of AC load flow of all the buses in the sample system.

\begin{tabular}{|c|c|c|c|c|c|c|c|c|c|}
\hline $\begin{array}{l}\text { Bus } \\
\text { No }\end{array}$ & $V(p u)$ & $\delta(\boldsymbol{R})$ & $\begin{array}{l}P_{g e n} \\
(\mathrm{pu})\end{array}$ & $\begin{array}{l}Q_{g e n} \\
(p u)\end{array}$ & $\begin{array}{c}\text { Bus } \\
\text { No }\end{array}$ & $V(p u)$ & $\delta(\boldsymbol{R})$ & $\begin{array}{l}\boldsymbol{P}_{\text {gen }} \\
(\mathrm{pu})\end{array}$ & $\begin{array}{l}Q_{g e n} \\
(p u)\end{array}$ \\
\hline 1 & 0,9550 & $\begin{array}{c}- \\
0,0326 \\
\end{array}$ & 0,5000 & 0,1882 & 60 & 0,9932 & $\begin{array}{c}- \\
0,1631 \\
\end{array}$ & - & - \\
\hline 2 & 0,9715 & $\begin{array}{c}- \\
0,0401\end{array}$ & - & - & 61 & 0,9950 & $\begin{array}{c}- \\
0,1496\end{array}$ & 1,0000 & $\begin{array}{c}- \\
0,3185\end{array}$ \\
\hline 3 & 0,9678 & $\begin{array}{c}- \\
0,0351\end{array}$ & - & - & 62 & 0,9980 & $\begin{array}{c}- \\
0,1523 \\
\end{array}$ & 0,5000 & $\begin{array}{c}- \\
0,1092 \\
\end{array}$ \\
\hline 4 & 0,9980 & $\begin{array}{c}- \\
0,0012 \\
\end{array}$ & 0,5000 & $\begin{array}{c}- \\
0,3627 \\
\end{array}$ & 63 & 0,9688 & $\begin{array}{c}- \\
0,1656 \\
\end{array}$ & - & - \\
\hline 5 & 1,0028 & 0,0007 & - & - & 64 & 0,9836 & $\begin{array}{c}- \\
0,1365 \\
\end{array}$ & - & - \\
\hline 6 & 0,9900 & $\begin{array}{c}- \\
0,0185 \\
\end{array}$ & 0,5000 & 0,0169 & 65 & 1,0050 & $\begin{array}{c}- \\
0,0788 \\
\end{array}$ & 2,0000 & 0,8208 \\
\hline 7 & 0,9893 & $\begin{array}{c}- \\
0,0265\end{array}$ & - & - & 66 & 1,0500 & $\begin{array}{c}- \\
0,1034\end{array}$ & 2,0000 & 0,1283 \\
\hline 8 & 1,0150 & 0,0428 & 0,5000 & $\begin{array}{c}- \\
0,2691 \\
\end{array}$ & 67 & 1,0200 & $\begin{array}{c}- \\
0,1396 \\
\end{array}$ & - & - \\
\hline 9 & 1,0500 & 0,0973 & - & - & 68 & 1,0032 & $\begin{array}{c}- \\
0,0699 \\
\end{array}$ & - & - \\
\hline 10 & 1,0500 & 0,1560 & 2,0000 & $\begin{array}{c}- \\
0,7807\end{array}$ & 69 & 1,0350 & 0,0000 & 4,5346 & $\begin{array}{c}- \\
0,5042\end{array}$ \\
\hline 11 & 0,9855 & $\begin{array}{c}- \\
0,0324 \\
\end{array}$ & - & - & 70 & 0,9840 & 0,0156 & 0,5000 & $\begin{array}{c}- \\
0,1494 \\
\end{array}$ \\
\hline 12 & 0,9900 & $\begin{array}{c}- \\
0,0331 \\
\end{array}$ & 1,0000 & 0,8467 & 71 & 0,9869 & 0,0444 & - & - \\
\hline 13 & 0,9687 & $\begin{array}{c}- \\
0,0519 \\
\end{array}$ & - & - & 72 & 0,9800 & 0,1112 & 0,5000 & $0,-\overline{2} 42$ \\
\hline 14 & 0,9836 & $\begin{array}{c}- \\
0,0426\end{array}$ & - & - & 73 & 0,9910 & 0,0647 & 0,5000 & 0,0042 \\
\hline 15 & 0,9700 & $\begin{array}{c}- \\
0,0394 \\
\end{array}$ & 0,5000 & $\begin{array}{c}- \\
0,0458 \\
\end{array}$ & 74 & 0,9580 & $\begin{array}{c}- \\
0,0318 \\
\end{array}$ & 0,5000 & $\begin{array}{c}- \\
0,1250 \\
\end{array}$ \\
\hline 16 & 0,9833 & $\begin{array}{c}- \\
0,0397 \\
\end{array}$ & - & - & 75 & 0,9686 & 0,0436 & - & - \\
\hline 17 & 0,9933 & $\begin{array}{c}- \\
0,0111\end{array}$ & - & - & 76 & 0,9430 & $\begin{array}{c}- \\
0,0703\end{array}$ & 0,5000 & $\stackrel{-}{0,1319}$ \\
\hline 18 & 0,9730 & $\begin{array}{c}- \\
0,0244 \\
\end{array}$ & 0,5000 & 0,1853 & 77 & 1,0060 & $\begin{array}{c}- \\
0,0802 \\
\end{array}$ & 0,5000 & 0,1428 \\
\hline 19 & 0,9620 & $\begin{array}{c}- \\
0,0345\end{array}$ & 0,5000 & $\begin{array}{c}- \\
0,2941\end{array}$ & 78 & 1,0015 & $\begin{array}{c}- \\
0,0898\end{array}$ & - & - \\
\hline 20 & 0,9588 & $\begin{array}{c}- \\
0,0335 \\
\end{array}$ & - & - & 79 & 1,0043 & 0,0931 & - & - \\
\hline 21 & 0,9606 & $\begin{array}{c}- \\
0,0162 \\
\end{array}$ & - & - & 80 & 1,0400 & $\begin{array}{c}- \\
0,0805 \\
\end{array}$ & 2,0000 & 1,7349 \\
\hline
\end{tabular}




\begin{tabular}{|c|c|c|c|c|c|c|c|c|c|}
\hline 22 & 0,9723 & 0,0164 & - & - & 81 & 0,9962 & $\begin{array}{c}- \\
0,0736 \\
\end{array}$ & - & - \\
\hline 23 & 1,0012 & 0,0834 & - & - & 82 & 0,9801 & $\begin{array}{c}- \\
0,0946\end{array}$ & - & - \\
\hline 24 & 0,9920 & 0,0978 & 0,5000 & $\begin{array}{c}- \\
0,2436 \\
\end{array}$ & 83 & 0,9767 & $\begin{array}{c}- \\
0,0823 \\
\end{array}$ & - & - \\
\hline 25 & 1,0500 & 0,1334 & 1,0000 & 0,5919 & 84 & 0,9775 & 0,0538 & - & - \\
\hline 26 & 1,0150 & 0,1484 & 2,0000 & $\begin{array}{c}- \\
0,0189 \\
\end{array}$ & 85 & 0,9850 & $\begin{array}{c}- \\
0,0344 \\
\end{array}$ & 0,5000 & $\begin{array}{c}- \\
0,0683 \\
\end{array}$ \\
\hline 27 & 0,9680 & 0,0374 & 0,5000 & $\begin{array}{c}- \\
0,1697 \\
\end{array}$ & 86 & 0,9898 & 0,0027 & - & - \\
\hline 28 & 0,9616 & 0,0154 & - & - & 87 & 1,0150 & 0,1038 & 0,5000 & 0,0570 \\
\hline 29 & 0,9631 & 0,0072 & - & - & 88 & 0,9893 & 0,0430 & - & - \\
\hline 30 & 0,9907 & 0,0161 & - & - & 89 & 1,0050 & $\begin{array}{c}- \\
0,0157 \\
\end{array}$ & 2,0000 & 0,4733 \\
\hline 31 & 0,9670 & 0,0124 & 0,5000 & 0,2063 & 90 & 0,9850 & $\begin{array}{c}- \\
0,0655\end{array}$ & 0,5000 & 0,4176 \\
\hline 32 & 0,9630 & 0,0351 & 0,5000 & $\begin{array}{c}- \\
0,3544 \\
\end{array}$ & 91 & 0,9800 & $\begin{array}{c}- \\
0,0304 \\
\end{array}$ & 0,5000 & $\begin{array}{c}- \\
0,2758 \\
\end{array}$ \\
\hline 33 & 0,9709 & $\begin{array}{c}- \\
0,0858 \\
\end{array}$ & - & - & 92 & 0,9900 & $\begin{array}{c}- \\
0,0398 \\
\end{array}$ & 0,5000 & $\begin{array}{c}- \\
0,3647 \\
\end{array}$ \\
\hline 34 & 0,9840 & $\begin{array}{c}- \\
0,1063 \\
\end{array}$ & 0,5000 & $\begin{array}{c}- \\
0,2759 \\
\end{array}$ & 93 & 0,9858 & $\begin{array}{c}- \\
0,0624 \\
\end{array}$ & - & - \\
\hline 35 & 0,9807 & $\begin{array}{c}- \\
0,1089\end{array}$ & - & - & 94 & 0,9889 & $\begin{array}{c}- \\
0,0741\end{array}$ & - & - \\
\hline 36 & 0,9800 & $\begin{array}{c}- \\
0,1062 \\
\end{array}$ & 0,5000 & $\begin{array}{c}- \\
0,0869 \\
\end{array}$ & 95 & 0,9781 & $\begin{array}{c}- \\
0,0917 \\
\end{array}$ & - & - \\
\hline 37 & 0,9921 & $0, \overline{1060}$ & - & - & 96 & 0,9885 & $0, \overline{-} \overline{53}$ & - & - \\
\hline 38 & 0,9694 & $\begin{array}{c}- \\
0,0608\end{array}$ & - & - & 97 & 1,0093 & $\begin{array}{c}- \\
0,0940\end{array}$ & - & - \\
\hline 39 & 0,9705 & $\begin{array}{c}- \\
0,1643 \\
\end{array}$ & - & - & 98 & 1,0234 & $\begin{array}{c}- \\
0,0879 \\
\end{array}$ & - & - \\
\hline 40 & 0,9700 & $\begin{array}{c}- \\
0,1828\end{array}$ & 0,5000 & 0,1216 & 99 & 1,0100 & $\begin{array}{c}- \\
0,0464\end{array}$ & 0,5000 & $\begin{array}{c}- \\
0,2875\end{array}$ \\
\hline 41 & 0,9668 & 0,2044 & 0,0000 & 0,0000 & 100 & 1,0170 & $0,-\overline{-} 333$ & 1,0000 & 1,2848 \\
\hline 42 & 0,9850 & $\begin{array}{c}- \\
0,2143 \\
\end{array}$ & 0,5000 & 0,1998 & 101 & 0,9927 & $\begin{array}{c}- \\
0,0570 \\
\end{array}$ & - & - \\
\hline 43 & 0,9704 & 0,1649 & - & - & 102 & 0,9901 & 0,0474 & - & - \\
\hline 44 & 0,9704 & 0,2063 & - & - & 103 & 1,0100 & 0,0200 & 0,5000 & 0,5789 \\
\hline 45 & 0,9782 & $\begin{array}{c}- \\
0,2071\end{array}$ & - & - & 104 & 0,9710 & $\begin{array}{c}- \\
0,0125\end{array}$ & 0,5000 & $\begin{array}{c}- \\
0,1276\end{array}$ \\
\hline 46 & 1,0050 & $\begin{array}{c}- \\
0,1707 \\
\end{array}$ & 0,5000 & 0,0329 & 105 & 0,9650 & $\begin{array}{c}- \\
0,0128 \\
\end{array}$ & 0,5000 & $\begin{array}{c}- \\
0,1532 \\
\end{array}$ \\
\hline 47 & 1,0173 & $\begin{array}{c}- \\
0,1611\end{array}$ & - & - & 106 & 0,9624 & $\begin{array}{c}- \\
0,0345\end{array}$ & - & - \\
\hline 48 & 1,0149 & 0,1778 & - & - & 107 & 0,9520 & $\begin{array}{c}- \\
0,0201\end{array}$ & 0,5000 & $\begin{array}{c}- \\
0,0441\end{array}$ \\
\hline 49 & 1,0250 & $\begin{array}{c}- \\
0,1714 \\
\end{array}$ & 1,0000 & 1,3987 & 108 & 0,9664 & $\begin{array}{c}- \\
0,0016 \\
\end{array}$ & - & - \\
\hline 50 & 1,0015 & 0,2010 & - & - & 109 & 0,9672 & 0,0036 & - & - \\
\hline
\end{tabular}




\begin{tabular}{|c|c|c|c|c|c|c|c|c|c|}
\hline 51 & 0,9677 & $\begin{array}{c}- \\
0,2393 \\
\end{array}$ & - & - & 110 & 0,9730 & 0,0233 & 0,5000 & $\begin{array}{c}- \\
0,0481 \\
\end{array}$ \\
\hline 52 & 0,9577 & $\begin{array}{c}- \\
0,2542\end{array}$ & - & - & 111 & 0,9800 & 0,0639 & 0,5000 & $\begin{array}{c}- \\
0,0540 \\
\end{array}$ \\
\hline 53 & 0,9464 & $\begin{array}{c}- \\
0,2665 \\
\end{array}$ & - & - & 112 & 0,9750 & 0,0085 & 0,5000 & 0,2021 \\
\hline 54 & 0,9550 & $\begin{array}{c}- \\
0,2471\end{array}$ & 0,5000 & 0,0191 & 113 & 0,9930 & 0,0064 & 0,5000 & $\begin{array}{c}- \\
0,0190\end{array}$ \\
\hline 55 & 0,9520 & $\begin{array}{c}- \\
0,2456 \\
\end{array}$ & 0,5000 & $\begin{array}{c}- \\
0,0980 \\
\end{array}$ & 114 & 0,9601 & 0,0263 & - & - \\
\hline 56 & 0,9540 & $\begin{array}{c}- \\
0,2461 \\
\end{array}$ & 0,5000 & $\begin{array}{c}- \\
0,2127 \\
\end{array}$ & 115 & 0,9600 & 0,0256 & - & - \\
\hline 57 & 0,9710 & $\begin{array}{c}- \\
0,2337 \\
\end{array}$ & - & - & 116 & 1,0050 & $\begin{array}{c}- \\
0,0755 \\
\end{array}$ & 0,5000 & 0,4698 \\
\hline 58 & 0,9595 & $\begin{array}{c}- \\
0,2474 \\
\end{array}$ & - & - & 117 & 0,9738 & $\begin{array}{c}- \\
0,0600 \\
\end{array}$ & - & - \\
\hline 59 & 0,9850 & $\begin{array}{c}- \\
0,2213 \\
\end{array}$ & 1,0000 & 0,8911 & 118 & 0,9501 & $\begin{array}{c}- \\
0,0640\end{array}$ & - & - \\
\hline \multicolumn{2}{|c|}{$\begin{array}{c}\text { Total Active } \\
\text { Power }\end{array}$} & 43,034601 & \multicolumn{2}{|c|}{ Total P Load } & \multicolumn{2}{|c|}{42,420000} & $P_{\text {loss }}$ & \multicolumn{2}{|c|}{0,614601} \\
\hline \multicolumn{2}{|c|}{$\begin{array}{c}\text { Total } \\
\text { Reactive } \\
\text { Power }\end{array}$} & 4,419482 & \multicolumn{2}{|c|}{$\begin{array}{c}\text { Total } Q \\
\text { Load }\end{array}$} & \multicolumn{2}{|c|}{14,380000} & $Q_{\text {loss }}$ & \multicolumn{2}{|c|}{$-9,961833$} \\
\hline \multicolumn{10}{|c|}{ Time (sec): 0,034583} \\
\hline
\end{tabular}

